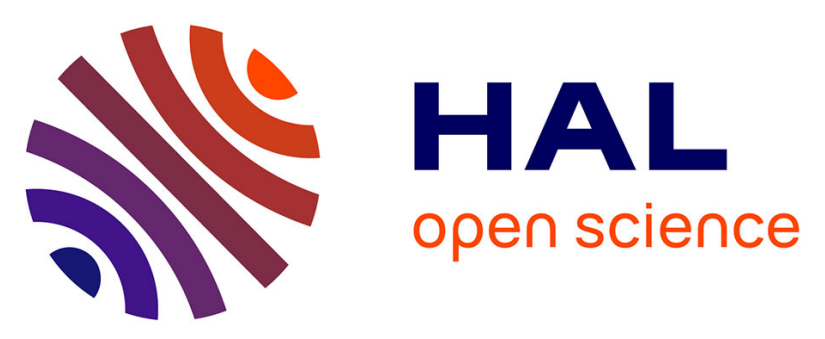

\title{
A New ER-MCDA Mapping for Decision-Making Based on Imperfect Information
}

\author{
Simon Carladous, Jean-Marc Tacnet, Jean Dezert, Guillaume Dupouy, \\ Mireille Batton-Hubert
}

\section{- To cite this version:}

Simon Carladous, Jean-Marc Tacnet, Jean Dezert, Guillaume Dupouy, Mireille Batton-Hubert. A New ER-MCDA Mapping for Decision-Making Based on Imperfect Information. Jiřina Vejnarová, Václav Kratochvíl. Belief Functions: Theory and Applications 4th International Conference, BELIEF 2016, Prague, Czech Republic, September 21-23, 2016, Proceedings, 9861 (5), Springer International Publishing, p. 46-55, 2016, 10.1007/978-3-319-45559-4_5 . hal-01408815v2

\section{HAL Id: hal-01408815 \\ https://hal.science/hal-01408815v2}

Submitted on 7 Dec 2016

HAL is a multi-disciplinary open access archive for the deposit and dissemination of scientific research documents, whether they are published or not. The documents may come from teaching and research institutions in France or abroad, or from public or private research centers.
L'archive ouverte pluridisciplinaire HAL, est destinée au dépôt et à la diffusion de documents scientifiques de niveau recherche, publiés ou non, émanant des établissements d'enseignement et de recherche français ou étrangers, des laboratoires publics ou privés. 


\title{
A New ER-MCDA Mapping for Decision-Making based on Imperfect Information
}

\author{
Simon Carladous ${ }^{1,2,4}$, Jean-Marc Tacnet ${ }^{1}$, Jean Dezert ${ }^{3}$, \\ Guillaume Dupouy ${ }^{1}$, and Mireille Batton-Hubert ${ }^{4}$ \\ 1 Université Grenoble Alpes, Irstea, UR ETGR, \\ 2 rue de la Papeterie-BP76, F-38402 St-Martin-d'Hères, France \\ \{simon.carladous, jean-marc.tacnet, guillaume.dupouy\}@irstea.fr \\ 2 AgroParisTech, 19 avenue du Maine, 75732 Paris, France \\ 3 The French Aerospace Lab, F-91761 Palaiseau, France \\ jean.dezert@onera.fr \\ 4 ENSMSE - DEMO, 29, rue Ponchardier 42100 Saint-Etienne, France \\ mbatton@emse.fr
}

\begin{abstract}
The Evidential Reasoning for Multi Criteria Decision Analysis (ER-MCDA) is based on a mapping process transforming a possibility distribution into a Bayesian basic belief assignment (BBA) related to a qualitative frame of discernement (FoD). Each element of the FoD is a fuzzy set. A new improved mapping method is proposed to get a final potentially non-Bayesian BBA on the FoD. We apply it to assess the stability of protective check dams against torrential floods given their imprecise scouring rate.
\end{abstract}

Keywords: fuzzy sets, possibility theory, belief functions, mapping, ERMCDA.

\section{Introduction}

Evidential Reasoning for Multi-Criteria Decision-Analysis (ER-MCDA) is a multicriteria decision method which is able to take into account both imperfect evaluation of quantitative and qualitative criteria and multiple more or less reliable sources [1]. The principle is first to represent imperfect evaluation of each criterion through a possibility distribution. As it uses fusion, those evaluations must be transformed into a common Frame of Discernment (FoD). Therefore, a mass of belief is assigned to consonant intervals with a confidence level which correspond to the possibility distribution. The mapping process establishes the link between the basic belief assignment BBA $m(\cdot)$ on quantitative intervals with a $\mathrm{BBA}$ in the common FoD. It can be interpreted as a function from the possibility distribution to a BBA on the common FoD. The initial principle of mapping is based on a geometrical projection. It induces several issues such as its theoretical justification but also its limited ability to provide positive masses of belief on singletons only. Our approach aims at improving this mapping process.

We consider an application case where ER-MCDA is used to assess the stability (high, medium, low) of check dams against torrential risk in mountains. 
This evaluation is based on several criteria, such as the scouring rate (expressed in percentage) which is a damageable loss of foundation support ${ }^{5}$ [2]. In this application context, the Fig. 1 shows how an expert often provides an imperfect evaluation of check dam stability according to its foundation's scouring rate. Indeed, a precise and direct measure would be somewhere too dangerous.

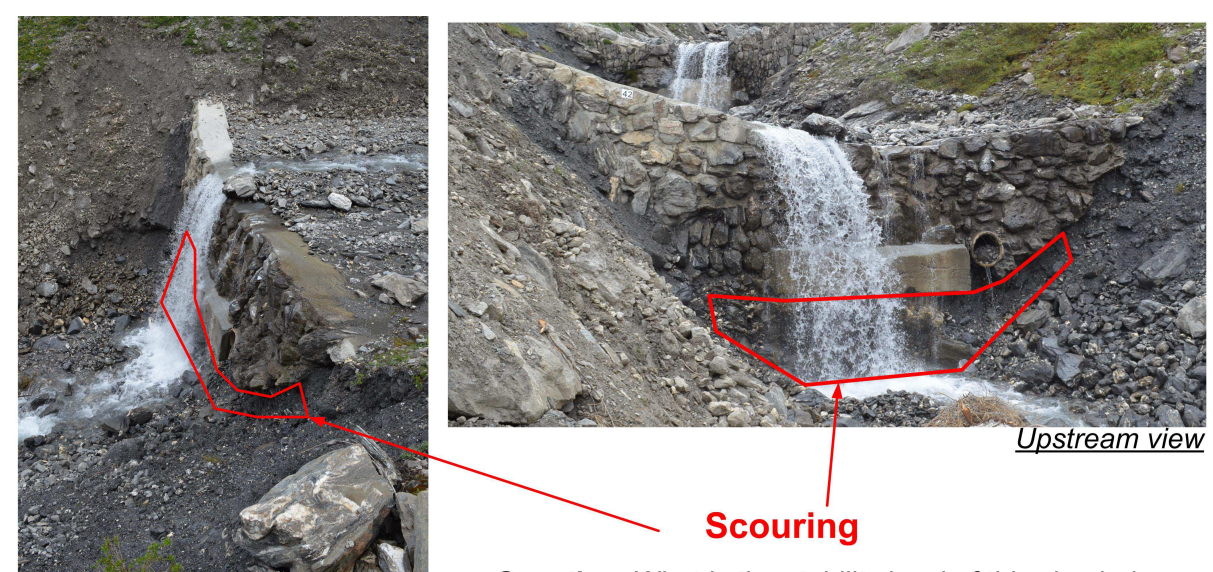

Question: What is the stability level of this check dam between 'high', 'medium', and 'low'?

- Expert answer - step 1: It depends on its foundation's scouring rate.

If it is between $0 \%$ and $30 \%$, it is completely 'high'. If it is between $50 \%$ and $60 \%$, it is completely 'medium'. If it is between $70 \%$ and $100 \%$, it is completely 'low'.

- Expert answer - step 2: It is too dangerous to measure the scouring rate precisely. I am certain it is between 40 and $60 \%$. I am not sure at all, but I think it is $50 \%$.

Expert - Expert answer - step 3: I think this check dam's stability is 'medium' but it could be 'medium' or 'high'. I am sure it is not 'low'.

Fig. 1. A real example of imperfect evaluation of check dam stability according to its foundation's scouring rate.

In this paper, our scope is not to develop a theoretical proof of the mapping process but to highlight some theoretical issues to propose another method. In section 2, we briefly recall some basics of fuzzy sets, possibility and belief function theories. We introduce the classical transformation and we propose a new method in section 3 . In section 4 , we compare the two methods on a sample decision context of our application case.

\footnotetext{
${ }^{5}$ Scouring is a process due to which the particles of the soil or rock under the check dam's foundation gets eroded and removed over a certain depth called scour depth and over the foundation area called scouring rate. Scouring often occurs in torrent because of the velocity and energy of the flowing in steep slopes.
} 


\section{Fuzzy sets, possibility and belief function theories}

In the fuzzy set theory [4], $U$ is the universe of discourse of individual elements $u$. $\mu_{A}$ is the membership function which associates each $u \in U$ to the class (fuzzy set) $A$ with the grade of membership $\mu_{A}(u) \in[0,1]$. A fuzzy set $A$ is normal when there is an element $u \in U$ such as $\mu_{A}(u)=1$. We use trapezoidal functions defined by the quadruplet $\{a, b, c, d\}$ (Eq. (1), Fig. 2) given their simplicity to approximate fuzzy intervals $[5]$. Intervals $[a, d]$ and $[b, c]$ are respectively the fuzzy set's support $\left(\operatorname{supp}_{A}\right)$ and its core $\left(A^{c}\right)[5]$. We denote $\bar{A}$ the complement of $A$ for $u \in U$, defined by the membership function $\mu_{\bar{A}}$ of the Eq. (2) and shown in the Fig. 2. Given $\mu_{B} \neq \mu_{A}$ which represents another fuzzy set $B$ for $u \in U$, the membership function $\mu_{A \cup B}$ of the Eq. (3) represents the union of $A$ and $B$ while $\mu_{A \cap B}$ of the Eq. (4) represents their intersection [4] (see Fig. 2).

$$
\mu_{A}(u) \triangleq\left\{\begin{array}{ll}
0 & \text { if } u \notin \operatorname{supp}_{A} \\
\frac{u-a}{b-a} & \text { if } u \in[a, b] \\
1 & \text { if } u \in A^{c}
\end{array} \quad(1) \quad \mu_{A \cup B}(u) \triangleq \max _{u \in U}\left(\mu_{A}(u), \mu_{B}(u)\right)\right.
$$

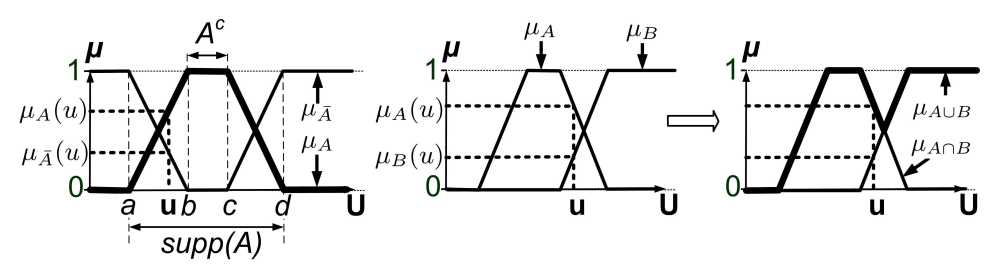

Fig. 2. Given $U$, trapezoidal fuzzy sets $A, \bar{A}, A \cup B$ and $A \cap B$.

In the possibility theory framework [6], $F$ is the fuzzy set of possible values of $u \in U$. The possibility distribution $\pi$ is given by $\mu_{F}(u) \triangleq \pi(u) \in[0,1]$ also defined by a quadruplet $\{a, b, c, d\}[7]$. Given $X$ a subset of $U$ and $\bar{X}$ its complement, the possibility measure is $\Pi(X) \triangleq \sup _{u \in X} \pi(u)[6]$ and the necessity is [8] $N(X) \triangleq 1-\Pi(\bar{X}), \forall X, \bar{X} \subseteq U$ as shown in the Fig. 3. Considering the fuzzy set $\mu_{A}$ and a possibility distribution $\mu_{F}$, the Eq. (5) gives the possibility measure of $A[6]$.

Considering $F$ as a nested family of $\alpha$-cuts $\left\{F_{\alpha} \mid \alpha \in(0,1]\right\}$, one has $F_{\alpha} \triangleq$ $\left\{u \mid \mu_{F}(u) \geqslant \alpha\right\}[5]$. Viewing $F_{\alpha}$ as a uniformly distributed random set from $\alpha \in(0,1]$ to $F_{\alpha} \subseteq U$, one has $\mu_{F}(u) \triangleq \int_{0}^{1} \mu_{F_{\alpha}}(u) \mathrm{d} \alpha$. Considering a finite nested family of subsets $\left\{F_{\alpha_{1}}=\right] u_{\alpha_{1}}^{-} ; u_{\alpha_{1}}^{+}\left[\subseteq \ldots \subseteq F_{\alpha_{l}}=\right] u_{\alpha_{l_{\max }}}^{-} ; u_{\alpha_{l_{\max }}^{+}}[\}$, the set $\left\{\alpha_{1}>\ldots>\alpha_{l}>\ldots>\alpha_{l_{\max }}\right\}$ and $m_{l} \triangleq \alpha_{l}-\alpha_{l+1} \in[0,1]$ with $\alpha_{l+1}=0$ (Fig. $3)$, one has the Eq. (6). 


$$
\Pi(A) \triangleq \sup _{u \in U} \mu_{A \cap F}(u)
$$

$$
\pi(u) \triangleq \sum_{u \in F_{\alpha_{l}}} m_{l}
$$

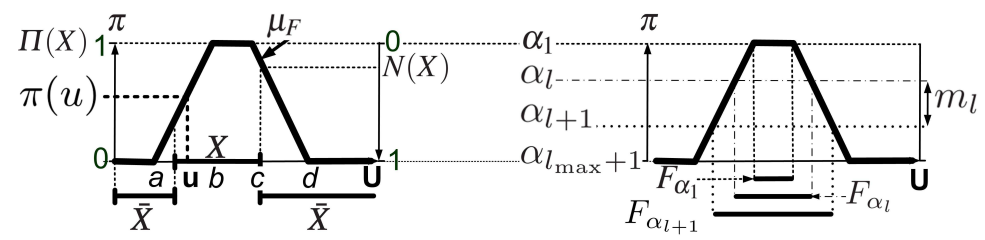

Fig. 3. Possibility distribution, possibility and necessity measures, $\alpha$-cuts.

In the belief function theory, a basic belief assignment (BBA), or mass of belief $m(\cdot)$, represents the imperfect knowledge of a body of evidence (or source) on a given FoD denoted as $\Theta=\left\{\theta_{1}, \theta_{2}, \ldots, \theta_{q}\right\}$. In the classical Shafer's model [9], all elements $\theta_{k}, k=1, \ldots, n$ are assumed exhaustive and mutually exclusive. The powerset $2^{\Theta}$ is the set of all subsets of $\Theta$, empty set $\emptyset$ included. For $X \in 2^{\Theta}$, we denote $\bar{X} \subseteq \Theta$ its complement. For each source, the mass function $m(\cdot): 2^{\Theta} \rightarrow$ $[0,1]$ must satisfy $m(\emptyset)=0$, and $\sum_{X \subseteq \Theta} m(X)=1, \forall X \neq \emptyset \in 2^{\Theta}$. Considering the universe of discourse $U$ as the FoD and assuming a normal fuzzy set $F, m(\cdot)$ is extracted considering $F_{\alpha_{l}} \in 2^{U}, l=1, \ldots, l_{\max }$.

Given $m(\cdot)$ on $2^{\Theta}$, the belief of the hypothesis $Y \in 2^{\Theta}$ is defined by $\operatorname{Bel}(Y) \triangleq$ $\sum_{X \subseteq Y \mid X \in 2^{\Theta}} m(X)$. Its plausibility is defined by $P l(Y) \triangleq \sum_{X \cap Y \neq \emptyset \mid X \in 2^{\Theta}} m(X)$ [10]. Considering that the universe of discourse $U$ is the $\operatorname{FoD} \Theta$, the plausibility measure $P l(X)$ is a possibility measure $\Pi(X), \forall X \subseteq \Theta=U[7]$.

Shafer's assumption of exhaustivity of the FoD means that it is considered as a "closed-world" (c.w.). In some practical problems, this assumption is too strict and it is more convenient to consider the original FoD as an "open-world" (o.w.). Dealing with it can be done in two manners as shown in the Fig. 4.

1. In Smets' Transferable Belief Model (TBM) [11], $\Theta^{\text {o.w. }} \triangleq\left\{\theta_{1}, \ldots, \theta_{q}\right\}$ and $\emptyset=\bar{\Theta}^{o . w .}$. One has $\sum_{X \in 2^{\Theta}} m(X)=1$, and one allows $m(\emptyset) \geqslant 0$.

2. In Yager's approach [12], the open-world is closed by an hedge element $\theta^{c}$, so that $\Theta^{\text {c.w. }} \triangleq \Theta^{\text {o.w. }} \cup\left\{\theta^{c}\right\}$. Setting $m^{\text {c.w. }}\left(\theta^{c}\right)=0$ and $m^{\text {c.w. }}(\emptyset)=0$, one has $\operatorname{Bel}^{\text {c.w. }}(A)=0$ and $m^{\text {c.w. }}(A)=0$ for each subset $A \subseteq \Theta^{o . w .}$. For each $X \subseteq \Theta^{c . w .}$, one computes $\mathrm{Bel}^{\text {c.w. }}(X)$ and $m^{\text {c.w. }}(X)$ in the Eqs. (7) and (8).

$$
\begin{gathered}
B e l^{c . w .}(X) \triangleq \begin{cases}0 & \text { if } X=A \subseteq \Theta^{o . w .} \subset \Theta^{c . w .} \\
1-P l^{c . w .}(\bar{X}) & \text { if } X=A \cup \theta^{c} \subseteq \Theta^{c . w .}\end{cases} \\
m^{\text {c.w. }}(X) \triangleq \begin{cases}0 & \text { if } X=A \subseteq \Theta^{\text {o.w. }} \subset \Theta^{\text {c.w. }} . \\
0 & \text { if } X=\theta^{c} \subset \Theta^{c . w .} \\
\sum_{Y \subseteq A}(-1)^{|A-Y|} \mathrm{Bel}^{\text {c.w. }}\left(Y \cup \theta^{c}\right) & \text { if } X=A \cup \theta^{c} \subseteq \Theta^{\text {c.w. }} .\end{cases}
\end{gathered}
$$




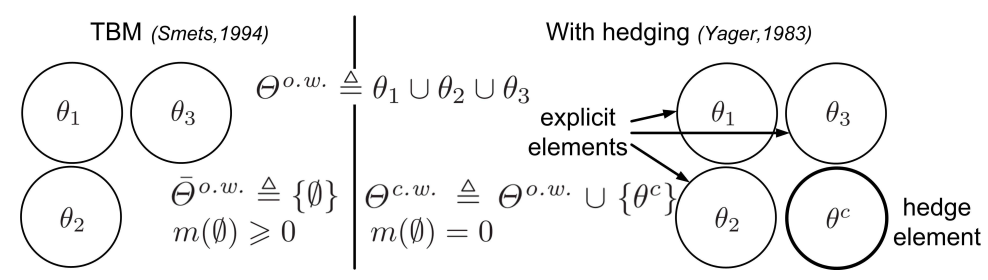

Fig. 4. TBM and hedging models under an "open-world" with exclusivity, for $q=3$.

\section{Transforming possibility distributions to BBAs on $\Theta$}

The $\operatorname{FoD} \Theta=\left\{\theta_{1}, \ldots, \theta_{q}\right\}$ of decision gathers $q$ qualitative labels. For each criterion, scoring results from an evaluation scale which is the specific universe of discourse $U$. To take into account its imprecise and uncertain evaluation, a possibility distribution $\pi: U \rightarrow[0,1]$ is given by the expert according to Eq.(6). To combine all the possibility distributions provided by several sources for several criteria, each one must be represented by a $\mathrm{BBA} m^{\Theta}(\cdot)$ established on $2^{\Theta}$ for the common FoD $\Theta$ as shown in the Fig. 5 .

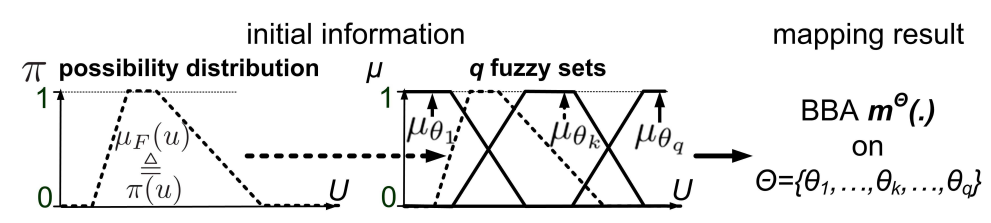

Fig. 5. The general principle and objective of the mapping process.

A mapping model [1] is therefore based on $q$ membership functions $\mu_{\theta_{k}}$ : $U \rightarrow[0,1]$ for $k=1, \ldots, q$ according to Eq.(1), corresponding to each $\theta_{k}$ of the FoD. The construction of the $q$ fuzzy sets respects the condition $\sum_{k=1}^{q} \mu_{\theta_{k}} \leqslant 1$ for mutual exclusivity: if an element $u^{\star} \in U$ totally belongs to a label $\theta_{k}$ $\left(\mu_{\theta_{k}}\left(u^{\star}\right)=1, k \in[1, q]\right)$, it cannot partially belongs to another label of $\Theta$ $\left(\mu_{\theta_{m}}\left(u^{\star}\right)=0, m \neq k \in[1, q]\right)$. By construction, there is at least $u^{\star} \in U$ for which $\mu_{\Theta=\bigcup\left\{\theta_{k} \mid k \in[1, q]\right\}}\left(u^{\star}\right) \leqslant 1$ : it corresponds to an "open-world" assumption.

\subsection{Classical transformation}

Given the possibility distribution $\pi$ and the $q$ functions $\mu_{\theta_{k}}$ for $k=1, \ldots, q$, the classical mapping [1] consists of the three following steps (shown in Fig. 6):

1. Given $l_{\max } \alpha$-cuts with $F_{\alpha_{l}} \in 2^{U}, l=1, \ldots, l_{\max }, m^{U}(\cdot)$ is extracted for the FoD $U$ from $\pi$ as shown in Fig. 3. Each $F_{\alpha_{l}}$ is a focal element and its corresponding mass is denoted $m^{U}\left(F_{\alpha_{l}}\right)$. The BBA $m^{U}(\cdot)$ thus represents the imperfect evaluation of a quantitative or qualitative evaluation of $u \in U$. 
Each focal element is an interval which represents evaluation imprecision. Each mass of an interval takes into account the confidence level or uncertainty. By definition of $m^{U}(\cdot)$, one has $\sum_{l=1}^{l_{\max }} m^{U}\left(F_{\alpha_{l}}\right)=1$.

2. For each $\left.F_{\alpha_{l}}=\right] u_{\alpha_{l}}^{-} ; u_{\alpha_{l}}^{+}\left[, l=1, \ldots, l_{\max }\right.$, the area $A_{k_{l}}$ under each curve $\mu_{\theta_{k}}$ is given by $A_{k_{l}} \triangleq \int_{u_{\alpha_{l}}^{-}}^{u_{\alpha_{l}}^{+}} \mu_{\theta_{k}}(u) d u$. One has $A_{l} \triangleq \sum_{k=1}^{q} A_{k_{l}}$ and computes the mass $m_{l}^{\Theta}\left(\theta_{k}\right) \triangleq \frac{A_{k_{l}}}{A_{l}}$ for each $F_{\alpha_{l}}$ and each $\theta_{k}$. Thus, by definition of $A_{l}$, one has $\sum_{k=1}^{q} m_{l}^{\Theta}\left(\theta_{k}\right)=1$.

3. One then builds the Bayesian ${ }^{6} \mathrm{BBA}^{\Theta}(\cdot)$ on $2^{\Theta}$ for the FoD $\Theta=\left\{\theta_{1}, \theta_{2}, \ldots, \theta_{q}\right\}$ with $m^{\Theta}\left(\theta_{k}\right) \triangleq \sum_{l=1}^{l_{\max }} m^{U}\left(F_{\alpha_{l}}\right) \times m_{l}^{\Theta}\left(\theta_{k}\right), k=1, \ldots, q$. This equation can be justified as follows.

From the two previous points, one has $\sum_{k=1}^{q} m_{l}^{\Theta}\left(\theta_{k}\right) \times \sum_{l=1}^{l_{\max }} m^{U}\left(F_{\alpha_{l}}\right)=1$. Thus $\sum_{k=1}^{q} \sum_{l=1}^{l_{\max }} m_{l}^{\Theta}\left(\theta_{k}\right) \times m^{U}\left(F_{\alpha_{l}}\right)=1$. By definition, $\sum_{k=1}^{q} m^{\Theta}\left(\theta_{k}\right)=1$. As a consequence, $m^{\Theta}\left(\theta_{k}\right) \triangleq \sum_{l=1}^{l_{\max }} m^{U}\left(F_{\alpha_{l}}\right) \times m_{l}^{\Theta}\left(\theta_{k}\right), k=1, \ldots, q$.

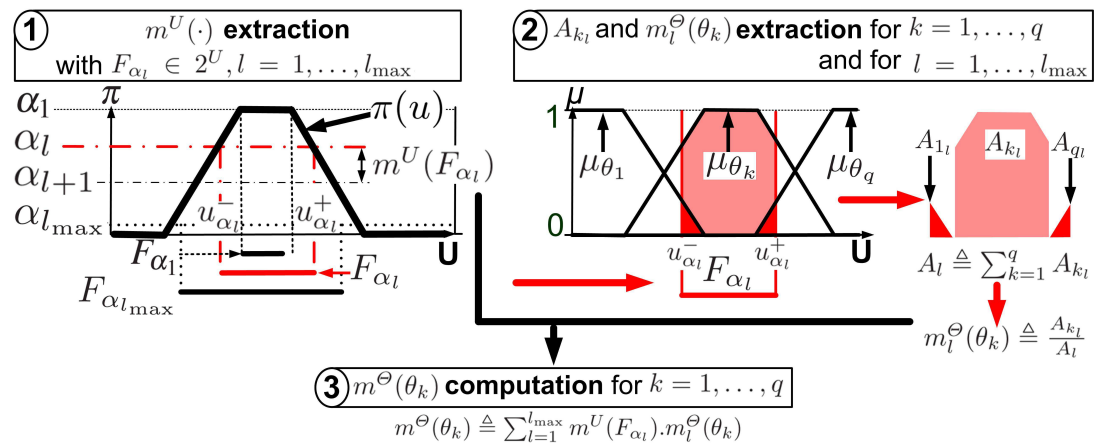

Fig. 6. Steps of the ER-MCDA's classical mapping process: $\alpha$-cuts choice, BBA $m^{U}(\cdot)$ calculation, and projection on the mapping model to get a final $\operatorname{BBA} m^{\Theta}(\cdot)$.

This principle of simple geometric transformation is however not fully satisfying since it only assigns BBAs to singletons and arbitrarily shares a BBA on several elements of the FoD regardless of partial or total ignorance. Moreover, from a practical point of view, this classical method needs an arbitrary setting of the value of $l_{\max }$ but also of all the values of $\alpha_{l}$. That is why, it was first proposed that experts give their imprecise evaluation through intervals with a confidence level. It corresponds to a possibility distribution after $\alpha$-cut, each $\alpha_{l}$ being the confidence level given by the expert [1]. Nevertheless, in practice, we think that it is easier to give only the quadruplet $\{a, b, c, d\}$ of Fig. 3 than several intervals with a confidence level.

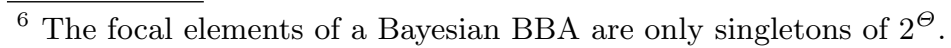




\subsection{New transformation}

Our new method is based on four steps to get a BBA $m^{\Theta}(\cdot)$ on all focal elements $X \in 2^{\Theta}$ and not only on singletons of $\Theta=\left\{\theta_{1}, \ldots, \theta_{k}, \ldots, \theta_{q}\right\}$. Given the construction of the $q$ fuzzy sets, it assumes mutual exclusivity without exhaustivity. Thereafter we detail the method using Yager's model with hedging even if a similar approach has been also studied using the TBM. One thus considers $\Theta^{o . w .}=\left\{\theta_{1}, \ldots, \theta_{k}, \ldots, \theta_{q}\right\}$ and $\Theta^{c . w .}=\Theta^{o . w .} \cup\left\{\theta^{c}\right\}$. Different steps are:

1. Construction of $2 q-1$ functions $\mu_{A \cup \dot{\theta}^{c}}^{c . w}$ for all $A \neq \emptyset \subseteq \Theta^{o . w}$. (see Fig. 7): given $q$ functions $\mu_{\theta_{k}}^{o . w}: U \rightarrow[0,1]$ for $k=1, \ldots, q$, the Eq. (3) gives the $2^{q}-1$ functions $\mu_{A}^{o . w .}: U \rightarrow[0,1]$. One uses the Eq. (9) to close the world and get the $2^{q}-1$ functions $\mu_{A \cup \theta^{c}}^{c . w}: U \rightarrow[0,1]$.

$$
\mu_{A \cup \theta^{c}}^{c . w}(u) \triangleq \begin{cases}1 & \text { if } \mu_{A}^{o . w} \cdot(u)=1 \\ \mu_{A}^{o . w} \cdot(u) & \text { if } \mu_{A}^{o \cdot w} \cdot(u)<1 \\ & \text { and if } \sum_{i=1}^{2^{q}} \mu_{Y_{i}}^{o . w} \cdot(u)>0, Y_{i} \subseteq \Theta^{o . w}, Y_{i} \cap A=\emptyset \\ 1 & \text { if } \mu_{A}^{o . w} \cdot(u)<1 \\ & \text { and if } \sum_{i=1}^{2^{q}} \mu_{Y_{i}}^{o . w} \cdot(u)=0, Y_{i} \subseteq \Theta^{o . w}, Y_{i} \cap A=\emptyset\end{cases}
$$

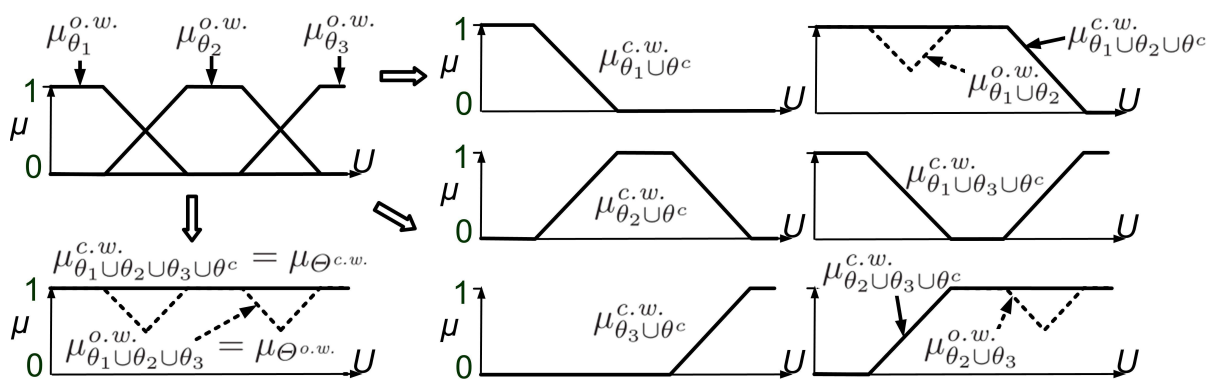

Fig. 7. Step 1 of the new mapping for $q=3$.

2. The Eq. (2) gives the $2^{q}-1$ functions $\mu \frac{c . w .}{A \cup \theta^{c}}: U \rightarrow[0,1]$. Given $\pi$ (or $\mu_{F}$ ), the Eq. (5) gives the possibility measures $\Pi^{c \cdot w} \cdot\left(\overline{A \cup \theta^{c}}\right) \triangleq \sup _{u \in U} \mu \overline{A \cup \theta^{c}} \cap F$, $\forall A \in 2^{\Theta^{o . w} \text {. }}-\{\emptyset\}$ corresponding to the plausibility measures $P l^{\text {c.w. }}\left(\overline{A \cup \theta^{c}}\right)$.

3. The Eq. (7) first provides $B e l^{c . w \cdot}(\cdot)$. The Eq. (8) then gives $m^{c . w \cdot}(\cdot)$ on $2^{\Theta^{c . w}}$. 


\section{Application to the scouring rate of a check dam}

In practice, the check dam stability is assessed through qualitative labels of the FoD $\Theta=\left\{\theta_{1}=\right.$ High, $\theta_{2}=$ Medium, $\theta_{3}=$ Low $\}$ [13]. The lower is the scouring rate, the higher is check dam structural stability. The FoD cardinality $|\Theta|$ is here $q=3$. For each check dam, the choice of the stability label notably depends on the scouring rate $u$ of its foundation, with $u$ a continuous value in $U=[0 \%, 100 \%] \cdot \mu_{\theta_{k}}$ is the membership function linking the scouring rate $u \in U$ to each stability label $\theta_{k}$. Each $\mu_{\theta_{k}}$ of the mapping model is represented in the Table 1 through a quadruplet $\{a, b, c, d\}$ according to the Eq. (1). The practical way of defining such a mapping model has been developed in [13]. It is based on a civil engineering analysis of the check dams stability given several hypotheses of scouring rate. They respect the exclusivity and "open-world" conditions given in the third paragraph of the section 3 .

In practice, experts imperfectly measure scouring rate as shown in Fig. 1. Therefore, we compare evaluations through possibility distributions provided by six different experts. Each evaluation is represented by a quadruplet $\{a, b, c, d\}$ in the Table 1 , from a very imprecise evaluation (expert 1 ) to very precise ones (experts 5 and 6 ). The evaluation illustrated in Fig. 1 is given by expert 3 .

Table 1. 3 fuzzy sets $\mu_{\theta_{k}}(u)$ and expert possibility distributions $\pi(u), \forall u \in U$.

\begin{tabular}{|c|c|c|c|c|c|c|}
\hline$\mu_{\theta_{k}}$ & $\theta_{1}$ & $\theta_{2}$ & $\theta_{3}$ & \multicolumn{3}{|c|}{} \\
$\mathrm{a}, \mathrm{b}, \mathrm{c}, \mathrm{d}$ & $0,0,30,50$ & $30,50,60,70$ & $60,70,100,100$ & & \\
\hline$\pi$ & Expert 1 & Expert 2 & Expert 3 & Expert 4 & Expert 5 & Expert 6 \\
a,b,c,d & $20,30,80,90$ & $30,40,60,80$ & $40,50,50,60$ & $30,40,40,50$ & $40,40,40,40$ & $45,45,45,45$ \\
\hline
\end{tabular}

To apply the classical transformation on the Table 1 , we assume $l_{\max }=4$ with $\alpha$-cuts $\alpha_{1}=1, \alpha_{2}=0.7, \alpha_{3}=0.4$, and $\alpha_{4}=0.1$ to extract the BBAs $m^{U}(\cdot)$ [10]. One gets the final Bayesian BBAs $m^{\Theta}(\cdot)$ in columns " 1 " in the Table 3.

After steps 1 and 2 using Table 1 values, one gets the values of $P l^{c . w} \cdot\left(\overline{A \cup \theta^{c}}\right)$, $\forall A \in 2^{\Theta^{o . w .}}-\{\emptyset\}$, with $\Theta^{\text {o.w. }}=\left\{\theta_{1}, \theta_{2}, \theta_{3}\right\}$. Using them in the step 4 , one gets the belief functions $\mathrm{Bel}^{\text {c.w. }}(\cdot)$ in the Table 2 and the BBAs $m^{\text {c.w. }}(\cdot)$ in columns " 2 " in the Table 3 . The latter gathers only focal elements with a positive mass.

Table 2. Intermediary $\mathrm{Bel}^{\text {c.w. }}\left(A \cup \theta^{c}\right)$ for the new mapping.

\begin{tabular}{|c|c|c|c|c|c|c|}
\hline$A \cup \theta^{c}$ & Expert 1 & Expert 2 & Expert 3 & Expert 4 & Expert 5 & Expert 6 \\
\hline$\theta_{1} \cup \theta^{c}$ & 0 & 0 & 0 & 0.333 & 0.5 & 0.25 \\
$\theta_{2} \cup \theta^{c}$ & 0 & 0.333 & 0.667 & 0.333 & 0.5 & 0.75 \\
$\theta_{1} \cup \theta_{2} \cup \theta^{c}$ & 0 & 0.333 & 1 & 1 & 1 & 1 \\
$\theta_{3} \cup \theta^{c}$ & 0 & 0 & 0 & 0 & 0 & 0 \\
$\theta_{1} \cup \theta_{3} \cup \theta^{c}$ & 0 & 0 & 0 & 0.333 & 0.5 & 0.25 \\
$\theta_{2} \cup \theta_{3} \cup \theta^{c}$ & 0 & 0.333 & 0.667 & 0.333 & 0.5 & 0.75 \\
$\theta_{1} \cup \theta_{2} \cup \theta_{3} \cup \theta^{c}$ & 1 & 1 & 1 & 1 & 1 & 1 \\
\hline
\end{tabular}


Results given in the Table 3 show that the new method affects a positive mass to total (experts 1 and 2) or partial ignorance with $m^{\text {c.w. }} \cdot\left(\theta_{1} \cup \theta_{2} \cup \theta^{c}\right)>0$ (experts 3 and 4 ) according to the imprecision degree of initial evaluations. The less precise is the initial evaluation of the scouring rate, the less informative is the mapped BBA. It improves the classical transformation and it propagates the imperfection of the initial information on $\Theta^{c . w}$.

Table 3. BBAs using two approaches: $1=m^{\Theta}(\cdot)$ for classical, and $2=m^{c \cdot w \cdot}(\cdot)$ for new.

\begin{tabular}{|c|c|c|c|c|c|c|c|c|c|c|c|c|}
\hline Focal & \multicolumn{2}{|c|}{ Expert 1 } & Expert 2 & Expert 3 & \multicolumn{3}{|c|}{ Expert 4 } & \multicolumn{3}{|c|}{ Expert 5} & \multicolumn{2}{|c|}{ Expert 6 } \\
elements & 1 & 2 & 1 & 2 & 1 & 2 & 1 & 2 & 1 & 2 & 1 & 2 \\
\hline$\theta_{1}$ & 0.24 & 0 & 0.15 & 0 & 0.05 & 0 & 0.5 & 0 & 0.5 & 0 & 0.25 & 0 \\
$\theta_{2}$ & 0.44 & 0 & 0.75 & 0 & 0.95 & 0 & 0.5 & 0 & 0.5 & 0 & 0.75 & 0 \\
$\theta_{3}$ & 0.32 & 0 & 0.10 & 0 & 0 & 0 & 0 & 0 & 0 & 0 & 0 & 0 \\
$\theta_{1} \cup \theta^{c}$ & - & 0 & - & 0 & - & 0 & - & $\frac{1}{3}$ & - & 0.5 & - & 0.25 \\
$\theta_{2} \cup \theta^{c}$ & - & 0 & - & $\frac{1}{3}$ & - & $\frac{2}{3}$ & - & $\frac{1}{3}$ & - & 0.5 & - & 0.75 \\
$\theta_{1} \cup \theta_{2} \cup \theta^{c}$ & - & 0 & - & 0 & - & $\frac{1}{3}$ & - & $\frac{1}{3}$ & - & 0 & - & 0 \\
$\theta_{1} \cup \theta_{2} \cup \theta_{3} \cup \theta^{c}$ & - & 1 & - & $\frac{2}{3}$ & - & 0 & - & 0 & - & 0 & - & 0 \\
\hline
\end{tabular}

\section{Conclusion}

This paper proposes a new mapping process in the ER-MCDA methodology. It allows to get a belief mass on non singletons elements of the powerset of the FoD. Unlike the initial approach [1], the possibility distribution is represented by its support and core and setting of values of $l_{\max }$ and $\alpha_{l}$ are not needed for an $\alpha$-cut approach. It is based on the conjunction between the possibility distribution and fuzzy sets but also takes into account the relationship between the possibility and the plausibility measures. To relax the hypothesis of exhaustivity of the classical Shafer's model, we have chosen Yager's hedging model instead of Smets' TBM model to prevent from introducing an ambiguity in the interpretation of the empty set. According to application cases, the hypothesis of exclusivity should be relaxed. It will be studied in forthcoming publications.

Acknowledgments. The authors thank the support of both French Ministry for Agriculture, Forest (MAAF) and Environment (MEEM). 


\section{References}

1. Tacnet, J.-M., Dezert, J., Batton-Hubert, M.: AHP and uncertainty theories for decision making using the ER-MCDA methodology. In: 11th Int. Symp. on AHP, Sorrento, Italy (2011)

2. Carladous, S., Tacnet, J.-M., Dezert, J., Batton-Hubert, M.: Belief function theory based decision support methods: Application to torrent protection work effectiveness and reliability assessment. In: 25th Int. Conf. ESREL, Zürich, Switzerland (2015)

3. Dezert, J., Tacnet, J.-M.: Evidential Reasoning for Multi-Criteria Analysis based on DSmT-AHP. In: 11th Int. Symp. on AHP, Sorrento, Italy (2011)

4. Zadeh, L.A.: Fuzzy sets. Inform. Control, vol. 8(3), pp. 338-353 (1965)

5. Dubois, D., Prade, H.: Fuzzy sets, probability and measurement. Eur. J. Oper. Res., vol. 40, pp. 135-154 (1989)

6. Zadeh, L.A.: Fuzzy sets as a basis for a theory of possibility. Fuzzy Set. Syst., vol. 1, pp. 3-28 (1978)

7. Dubois, D., Prade, H.: Properties of measures of information in evidence and possibility theories. Fuzzy Set. Syst., vol. 24, pp. 161-182 (1987)

8. Dubois, D., Prade, H.: Possibility Theory - An approach to Competurized Processing of Uncertainty. Springer (1988)

9. Shafer, G.: A Mathematical Theory of Evidence. Princeton University Press (1976)

10. Baudrit, C., Guyonnet, D., Dubois, D.: Postprocessing the hybrid method for addressing uncertainty in risk assessments. J. Environ. Eng., vol. 131, pp. 1750-1754 (2005)

11. Smets, P., Kennes, R.: The transferable belief model. Artif. Intell., vol. 66, pp. 191-234 (1994)

12. Yager, R.R.: Hedging in the Combination of Evidence. J. Inform. Sci., vol. (1), pp. 73-81 (1983)

13. Carladous, S., Tacnet, J.-M., Di Ciocco, G., Curt, C., Batton-Hubert, M.: Approche quantiative pour élaborer une échelle d'évaluation floue d'un indicateur de stabilité structurale. Application l'affouillement d'ouvrages de stabilisation torrentielle. In: 9th Conf. JFMS, Nancy, France (2016) 